\title{
Comparison of Risk Assessment Strategies for Patients with Diabetes Mellitus and Stable Chest Pain: A Coronary Computed Tomography Angiography Study
}

\author{
Jia Zhao, ${ }^{1,2}$ Shuo Wang, ${ }^{3}$ Pengyu Zhao, ${ }^{4}$ Yong Huo, ${ }^{5}$ Chunjie Li $\mathbb{D}^{1}$ and Jia Zhou $\mathbb{D}^{1}$ \\ ${ }^{1}$ Department of Cardiology, Tianjin Chest Hospital, Tianjin, China \\ ${ }^{2}$ Graduate School, Tianjin Medical University, Tianjin, China \\ ${ }^{3}$ Graduate School, Tianjin University of Traditional Chinese Medicine, Tianjin, China \\ ${ }^{4}$ School of Electrical and Information Engineering, Tianjin University, Tianjin, China \\ ${ }^{5}$ Department of Cardiology, Peking University First Hospital, Beijing, China
}

Correspondence should be addressed to Chunjie Li; ykwn@sina.com and Jia Zhou; zhoujiawenzhang@126.com

Received 5 October 2021; Revised 14 December 2021; Accepted 7 January 2022; Published 25 January 2022

Academic Editor: Pawel Kleczynski

Copyright ( 2022 Jia Zhao et al. This is an open access article distributed under the Creative Commons Attribution License, which permits unrestricted use, distribution, and reproduction in any medium, provided the original work is properly cited.

\begin{abstract}
Background. To compare two risk assessment strategies to identify individuals likely to benefit from further imaging testing in patients with diabetes mellitus (DM) and stable chest pain (SCP) suspected of obstructive coronary artery disease (CAD). Methods. 602 DM patients referred to coronary computed tomography angiography (CCTA) for SCP were included. They were divided into high- and low-risk groups according to the 2016 National Institute of Health and Care Excellence guidelinedetermined strategy (NICE strategy) which focused on symptom evaluation and 2019 European Society of Cardiology guideline-determined strategy (ESC strategy) which was based on pretest probability (PTP) sequentially determined by the ESC-PTP estimator and risk factor-weighted clinical likelihood (RF-CL) model, respectively. The associations of clinical outcomes with risk groups and net reclassification improvement (NRI) were evaluated. Results. The NICE and ESC strategy classified $44 \%$ and $39 \%$ patients into the low-risk group, respectively. Compared to the NICE strategy, the ESC strategy indicated stronger associations between risk groups and events (hazard ratios: 4.24 versus 1.91), intensive clinical management, and a positive NRI $(27.71 \%, p<0.0001)$. The application of the RF-CL model ameliorated the underestimation of risk in patients with borderline ESC-PTP, which principally account for the improvement of the ESC strategy. Conclusion. Compared to the NICE strategy, the ESC strategy seemed to be associated with greater efficiency in identifying high risk individuals in patients with DM and SCP.
\end{abstract}

\section{Introduction}

In patients with diabetes mellitus (DM), coronary artery disease (CAD) is a major cause of mortality [1]. Stable chest pain (SCP) is the most common clinical manifestation in patients with obstructive CAD. In an analysis for the largest contemporary cohort of SCP, the PROMISE trial, patients with DM were more likely to have a positive cardiovascular imaging testing (CIT) result and major adverse cardiovascular event (MACE) [2]. Consequently, a risk assessment strategy to efficiently identify high-risk individuals deriving maximum benefit from further CIT is initial and essential in the clinical management for DM patients presenting with SCP suggestive of obstructive CAD [2-4].

To improve this identification, the 2016 U.K. National Institute of Health and Care Excellence (NICE) guideline recommended a symptom-based risk assessment strategy for SCP [5]. However, this strategy has been controversial since its release [6-8] and numerous studies have indicated that atypical symptoms were more likely to be a manifestation in patients with $\mathrm{DM}[2,3,9]$. On the other hand, the 2019 European Society of Cardiology (ESC) guideline advocated an updated pretest probability (PTP) estimator based on age, sex, and symptom and recommended CIT for 
patients with high ESC-PTP [10]. For patients with borderline ESC-PTP, the addition of other risk factors can improve the estimation of clinical likelihood of obstructive CAD [11].

The 2016 NICE guideline-determined risk assessment strategy (NICE strategy) [12,13] and ESC-PTP estimator [14-16] have been externally validated in general SCP patients. But to date, no comparative analysis has been conducted to systematically evaluate the NICE strategy and 2019 ESC guideline-determined risk assessment strategy (ESC strategy) in patients with both DM and SCP, for whom the appropriate referral for intensive investigation was fundamental but difficult $[2,3,17]$. Thus, we aimed to compare the two newest risk assessment strategies to optimize decision-making of downstream clinical management in a coronary computed tomography angiography- (CCTA-) based cohort comprised of patients with DM and SCP.

\section{Methods}

2.1. Study Population. As described previously, 5289 patients referred to CCTA for SCP indicative of obstructive CAD were recruited from December 2015 to December 2017 in Tianjin Chest Hospital [18-21]. In the present analysis, 602 patients with a diagnosis of DM were included and followed up until December 2019. Patients were considered suffering from DM if one of the following was met: treatment with insulin or hypoglycemic medications, fasting blood glucose $\geq 7.0 \mathrm{mmol} / \mathrm{L}$, a $2 \mathrm{~h}$ plasma glucose level on their oral glucose tolerance test $\geq 11.1 \mathrm{mmol} / \mathrm{L}$, or a glycated hemoglobin value $\geq 6.5 \%$. This observational study was conducted after obtaining the informed consent from the participating patients and upon the approval by the ethics committee of Tianjin Chest Hospital.

2.2. Baseline Data. Baseline data such as age, sex, hypertension, hyperlipidemia, smoking, abnormal electrocardiograph, creatinine, and symptom were collected and defined as described previously [18-21]. SCP symptom was categorized as nonanginal chest pain, atypical angina, or typical angina [22]. For each patient, creatinine was routinely measured unless the measurement has happened within 2 months before CCTA. The estimated glomerular filtration rate was calculated based on the CKD-EPI formula [23].

2.3. Risk Assessment Strategies. A patient in the high-risk group based on each strategy should take CIT. Details of risk groups in the NICE and ESC strategy were as follows $[5,10]$ :

NICE strategy: patients with nonanginal SCP and normal ECG were at low risk. The high-risk group included SCP patients who were diagnosed with typical and atypical angina or nonanginal pectoris with abnormal ECG [5].

ESC strategy: PTP of obstructive CAD was determined according to the ESC-PTP estimator based on age, sex, and symptom [10]. Patients with ESC-PTP $<5 \%$ were divided into the low-risk group, and patients with ESC-PTP $>15 \%$ were divided into the high-risk group. For other patients, we used the risk factor-weighted clinical likelihood (RFCL) model for further assessment [24]. The RF-CL model incorporating clinical variables plus age, sex, and symptom showed the most robust performance. According to the data from the original study of the RF-CL model, low RF-CL $(<15 \%)$ was associated with less obstructive CAD $(<5 \%)$ and risk of clinical events ( $<2 \%$ annual risk) [24]. Thus, patients with ESC-PTP between $5 \%$ and $15 \%$ and RF-CL $<$ $15 \%$ were at low risk, and patients with ESC-PTP between $5 \%$ and $15 \%$ and RF-CL $>15 \%$ were at high risk.

2.4. CCTA. All scans were performed according to the established guideline [25] and institutional protocols [18-21]. In image evaluation, each coronary segment with a $>2 \mathrm{~mm}$ diameter was analyzed for the presence of coronary diameter stenosis. According to the Coronary Artery DiseaseReporting and Data System [26], the maximal degree of coronary diameter stenosis was defined as $0 \%, 1-49 \%$, and $50 \%$. Obstructive CAD was defined as present if a patient had at least one lesion with $\geq 50 \%$ diameter stenosis or any unassessable segments at CCTA. The patient with obstructive CAD was defined as positive.

2.5. Follow-Up and Clinical Events. After CCTA, all patients were followed at $6,12,24,36$, and 48 months by phone call or physician visit. MACE, defined as cardiac death and myocardial infarction, was the primary endpoint. Cardiac death was defined as any death caused by cardiac disease or for which no other cause could be found. Myocardial infarction was defined as described in the Fourth Universal Definition of Myocardial Infarction [27]. The changes of downstream clinical management, which included medication prescriptions (such as antiplatelet agents, anti-ischemic drugs, and lipid-lowering agents), referrals to CIT (noninvasive and invasive imaging testing), and coronary revascularization (CR) within 60 days after CCTA, were identified on an electronic medical system. Increase of medication (IM), invasive coronary angiography (ICA), and CR were regarded as secondary endpoints. All endpoints were adjudicated via review of follow-up information and medical records by an independent clinical event committee who were blinded to other data.

2.6. Statistical Analysis. Student's $t$-test or Mann-Whitney $U$ test was used to evaluate the differences in continuous variables appropriately. The $\chi^{2}$ test or Fisher exact test was used to evaluate the differences in categorical variables appropriately. All statistical analyses were performed using MedCalc (version 15.2.2; MedCalc Software, Mariakerke, Belgium) and R (version 3.2.4; R Foundation for Statistical Computing, Vienna, Austria). The discrimination and calibration of the ESC-PTP estimator were assessed by the area under receiver operating characteristic curve (AUC) and Hosmer-Lemeshow goodness-of-fit statistic (H-L $\left.\chi^{2}\right)$ according to the Transparent Reporting of a Multivariable Prediction Model for Individual Prognosis or Diagnosis (TRIPOD) statement [28]. Net reclassification improvement (NRI) was assessed in a reclassification table and used to determine how a risk assessment strategy reclassified patients into various risk groups compared with another [29]. The cumulative MACE-free survivals were estimated using KaplanMeier curves and were compared by the log-rank test. We 
used Cox proportional hazards regression models to calculate hazard ratios (HRs) and 95\% confidence intervals (CIs). Two-tailed $p<0.05$ was considered statistically significant.

\section{Results}

3.1. Baseline Characteristics. The study cohort consisted of 602 DM patients, of whom $45.02 \%$ (271/602) were found to have obstructive CAD on CCTA which are listed in Table 1. Most baseline characteristics were significantly associated with the presence of obstructive CAD. According to the NICE strategy, of the 602 patients, $43.68 \%$ (263/602) were assigned to the low-risk group. There were 87 patients with a RF-CL $<15 \%$ among 208 patients with an ESC-PTP of 5-15\%. Together with the 150 patients with an ESC-PTP below 5\%, the ESC strategy totally classified $39.37 \%$ (237/ 602) into the low-risk group.

Table 2 shows the distribution of clinical characteristics by risk groups based on different strategies. Except hypertension and hyperlipidemia, differences of the other baseline characteristics were statistically significant between two risk groups based on the NICE strategy. There were significant differences in most characteristics except abnormal ECG in terms of the ESC strategy. Compared with low-risk patients, high-risk patients had more obstructive CAD (NICE strategy: $61 \%$ versus $25 \%, p<0.0001$; ESC strategy: $71 \%$ versus $5 \%, p<0.0001$ ) and MACE (NICE strategy: $9 \%$ versus $5 \%$, $p=0.0422$; ESC strategy: $10 \%$ versus $3 \%, p=0.0001$ ).

3.2. Follow-Up. Patients were followed up for a median of 36 (interquartile range: 30 to 43 ) months, and 37 patients experienced MACE (8 cardiac deaths and 29 nonfatal MI). Figure 1 illustrates the Kaplan-Meier estimates of patients surviving free from MACE. The high-risk group according to both NICE and ESC strategies had a significantly higher risk of MACE, respectively ( $p$ for the log-rank test: 0.0445 for the NICE strategy and 0.0003 for the ESC strategy), but the association of ESC strategy-determined risk groups (high versus low) with MACE was stronger than that of the NICE strategy (HR for NICE strategy: 1.91, 95\% CI 1.01-3.63, $p$ $=0.0485$; HR for ESC strategy: 4.24, 95\% CI 1.80-9.97, $p=$ $0.0010)$.

3.3. Subsequent Clinical Management. The associations between risk groups and secondary endpoints according to the NICE and ESC strategy are manifested in Figure 2. 175 patients had ICA based on CCTA, 138 patients had obstructive CAD on ICA, and 65 patients underwent CR. Compared with low-risk patients, high-risk patients had more IM (NICE strategy: 48\% (164/339) versus 29\% (77/263), odds ratio (OR): 2.26, 95\% CI: 1.61-3.18, $p<0.0001$; ESC strategy: $53 \%$ (195/365) versus $19 \%$ (46/237), OR: $4.76,95 \%$ CI: $3.25-$ $6.98, p<0.0001)$, ICA (NICE strategy: $37 \%(125 / 339)$ versus 19\% (49/263), OR: 2.55, 95\% CI: 1.74-3.73, $p<0.0001$; ESC strategy: $40 \%(145 / 365)$ versus $12 \%(29 / 237)$, OR: 4.73 , 95\% CI: 3.04-7.35, $p<0.0001$ ), and CR (NICE strategy: $13 \%(44 / 339)$ versus $8 \%$ (20/263), OR: 2.03 , 95\% CI: $1.14-$
3.60, $p=0.0156$; ESC strategy: $16 \%(59 / 365)$ versus $2 \%(5 /$ 237), OR: $8.89,95 \%$ CI: $3.51-22.50, p<0.0001)$.

3.4. Validation of the ESC-PTP Estimator. The receiver operating characteristic curves of the ESC-PTP estimator are illustrated in Figure 3. The discrimination of the ESC-PTP estimator was acceptable, with an AUC of 0.783 (95\% CI 0.747 to $0.819, p<0.0001)$. The calibration plot for the ESC-PTP estimator is presented in Figure 4. Graphically, the ESC-PTP estimator underestimated the probability of obstructive CAD in patients with an ESC-PTP between 5\% and $15 \%$ and overestimated the probability of obstructive CAD in patients with an ESC-PTP $>15 \%$, resulting in a poor calibration $\left(\mathrm{H}-\mathrm{L} \chi^{2}=92.47, p<0.0001\right)$.

3.5. Comparison of the ESC Strategy and NICE Strategy by NRI. Table 3 is the reclassification table comparing the ESC strategy to the NICE strategy. Compared to the NICE strategy, among the 331 negative patients, 35 patients were correctly reclassified from high- to low-risk groups by the ESC strategy, but 8 negative patients were incorrectly reclassified from low- to high-risk groups by the ESC strategy. Among the 271 positive patients, the ESC strategy correctly reclassified 59 patients from low- to high-risk groups, but 6 patients were incorrectly reclassified from high- to low-risk groups. Therefore, the NRI of the ESC strategy compared with the NICE strategy was $8.15 \%$ for negative patients, $19.66 \%$ for positive patients, and $27.71 \%$ for all patients. Table 4 is the reclassification table comparing the RF-CL model to the NICE strategy in patients with ESC-PTP between $5 \%$ and $15 \%$. Compared to the NICE strategy, the RF-CL model correctly reclassified 36 positive patients into the high-risk group, in large measure accounting for the NRI of $32.29 \%$ in positive and the NRI of $42.11 \%$ in all. As shown in Table 5, the improvement was attenuated when the analysis was applied to patients with ESC-PTP below $5 \%$ and above $15 \%$, either comparing the RF-CL model to the NICE strategy $(\mathrm{NRI}=24.33 \%, p<0.0001)$ or comparing the ESC strategy to the NICE strategy (NRI $=19.88 \%, p<$ 0.0001 ), resulting from the similar classification of the ESC strategy and RF-CL model (NRI $=-4.45 \%, p=0.0598$ ).

\section{Discussion}

In this CCTA-based cohort comprised of patients with DM and SCP, based on two newest risk assessment strategies, low-risk groups were associated with fewer obstructive CAD, MACE, and clinical interventions than high-risk groups did. Compared to the NICE strategy which focused on symptom evaluation, the ESC strategy which sequentially incorporated the ESC-PTP estimator with the RF-CL model had more potential to optimize decision-making of downstream referral for CIT in patients with DM and SCP.

It has been well established that DM confers a two-fold increased risk of MACE in patients presenting with SCP potentially related to CAD [2]. Thus, the referral of CIT to screening for obstructive CAD guided by the risk assessment strategy is vital in the clinical management of patients with DM and SCP, but the most efficient strategy for these 
TABLE 1: Baseline characteristics by the presence of obstructive CAD on CCTA.

\begin{tabular}{|c|c|c|c|c|}
\hline \multirow{2}{*}{ Characteristic } & \multirow{2}{*}{$\begin{array}{c}\text { Total } \\
N=602\end{array}$} & \multicolumn{2}{|c|}{ Obstructive CAD } & \multirow[b]{2}{*}{$p$} \\
\hline & & Yes $(N=271)$ & No $(N=331)$ & \\
\hline Age (years, mean $\pm \mathrm{SD}$ ) & $62.26 \pm 11.61$ & $65.83 \pm 12.79$ & $59.34 \pm 11.96$ & $<0.0001$ \\
\hline Male & $331(55)$ & $176(65)$ & $155(47)$ & $<0.0001$ \\
\hline Hypertension & $409(68)$ & $198(73)$ & $211(64)$ & 0.0188 \\
\hline Hyperlipidemia & $313(52)$ & $160(59)$ & $153(46)$ & 0.0023 \\
\hline Smoking & $284(47)$ & $143(53)$ & $141(43)$ & 0.0162 \\
\hline Abnormal ECG & $259(43)$ & $132(49)$ & $127(38)$ & 0.0136 \\
\hline $\mathrm{eGFR}\left(\mathrm{mL} / \mathrm{min} / 1.73 \mathrm{~m}^{2}\right.$, mean $\left.\pm \mathrm{SD}\right)$ & $71.59 \pm 9.47$ & $70.34 \pm 10.49$ & $72.61 \pm 12.07$ & 0.0152 \\
\hline Symptom & & & & 0.0182 \\
\hline Nonanginal chest pain & $284(47)$ & $115(42)$ & $169(51)$ & \\
\hline Atypical angina & $239(40)$ & $110(41)$ & $129(39)$ & \\
\hline Typical angina & $79(13)$ & $46(17)$ & $33(10)$ & \\
\hline
\end{tabular}

SD: standard deviation; CAD: coronary artery disease; ECG: electrocardiogram; CCTA: coronary computed tomographic angiography; eGFR: estimated glomerular filtration rate. Values are presented as $n(\%)$ unless stated otherwise.

TABLE 2: Characteristics by risk groups based on the NICE and ESC strategy.

\begin{tabular}{|c|c|c|c|c|c|c|c|}
\hline & \multirow[b]{2}{*}{$\begin{array}{c}\text { Total } \\
n=602\end{array}$} & \multicolumn{3}{|c|}{ NICE strategy } & \multicolumn{3}{|c|}{ ESC strategy } \\
\hline & & $\begin{array}{c}\text { Low } \\
n=263\end{array}$ & $\begin{array}{c}\text { High } \\
n=339\end{array}$ & $p$ & $\begin{array}{c}\text { Low } \\
n=237\end{array}$ & $\begin{array}{c}\text { High } \\
n=365\end{array}$ & $p$ \\
\hline Age (years, mean $\pm S D$ ) & $62.26 \pm 11.61$ & $59.99 \pm 12.53$ & $64.02 \pm 12.10$ & $<0.0001$ & $57.44 \pm 12.44$ & $65.39 \pm 12.27$ & $<0.0001$ \\
\hline Female & $331(55)$ & $128(49)$ & $203(60)$ & 0.0078 & $101(43)$ & $230(63)$ & $<0.0001$ \\
\hline Hypertension & $409(68)$ & $172(65)$ & $237(70)$ & 0.2763 & $143(60)$ & $266(73)$ & 0.0017 \\
\hline Hyperlipidemia & $313(52)$ & $130(49)$ & $183(54)$ & 0.3045 & $109(46)$ & $204(56)$ & 0.0219 \\
\hline Smoking & $284(47)$ & $111(42)$ & $173(51)$ & 0.0385 & $91(38)$ & $193(53)$ & 0.0007 \\
\hline Abnormal ECG & $259(43)$ & $0(0)$ & $259(76)$ & $<0.0001$ & $95(40)$ & $164(45)$ & 0.2760 \\
\hline Symptom & & & & $<0.0001$ & & & 0.0001 \\
\hline Nonanginal chest pain & $284(47)$ & $263(100)$ & $21(6)$ & & $91(38)$ & $193(53)$ & \\
\hline Atypical angina & $239(40)$ & $0(0)$ & $239(71)$ & & $100(42)$ & $139(38)$ & \\
\hline Typical angina & 79 (13) & $0(0)$ & $79(23)$ & & $46(20)$ & $33(9)$ & \\
\hline Obstructive $\mathrm{CAD}^{\mathrm{b}}$ & $271(45)$ & $65(25)$ & $206(61)$ & $<0.0001$ & $12(5)$ & $259(71)$ & $<0.0001$ \\
\hline MACE & $45(7)$ & $13(5)$ & $32(9)$ & 0.0422 & $6(3)$ & $39(10)$ & 0.0001 \\
\hline Cardiac death & $11(2)$ & $2(1)$ & $9(3)$ & 0.1244 & $0(0)$ & $11(3)$ & 0.0044 \\
\hline Nonfatal MI & $34(5)$ & $11(4)$ & $23(6)$ & 0.2325 & $6(3)$ & $28(7)$ & 0.0067 \\
\hline
\end{tabular}

SD: standard deviation; CAD: coronary artery disease; NICE strategy: 2016 National Institute of Health and Care Excellence guideline-determined risk assessment strategy; ESC strategy: 2019 European Society of Cardiology guideline-determined risk assessment strategy; ECG: electrocardiogram; MI: myocardial infarction; MACE: major adverse cardiovascular events. Values are presented as $n$ (\%) unless stated otherwise.

patients has been debated until now $[2,3]$. In stark contrast to the "screen all" strategy which was not supported by contemporary evidence $[17,30]$, the NICE strategy recommended a simple evaluation based on symptom [5] and improved diagnostic certainty and clinical outcomes compared to traditional PTP calculator-based strategies in two external validation studies conducted in general SCP patients $[12,13]$. However, compared to the ESC strategy, the NICE strategy was demonstrated to be less efficient in the risk assessment for patients with DM and SCP.

Because of autonomic neuropathy affecting the pain perceptual threshold, the association between ischemia and SCP may be diminished, which could lead to an atypical presen- tation in patients with $\mathrm{DM}[9,31,32]$. As a result, current evidence implied that the suboptimal performance of the NICE strategy may, to a large extent, be attributed to the insufficient power of symptom evaluation alone in patients with DM and SCP. In the DM subgroup of the PROMISE cohort, neither typical nor atypical chest pain was an independent predictor of positive CIT [2]. Meanwhile, an analysis conducted in 8662 patients referred for new-onset SCP suggested that among patients diagnosed with noncardiac chest pain, those with DM remained at two-fold increased risk of MACE, compared with non-DM patients [3]. In conformity with these findings, when comparing the NICE strategy to the ESC strategy in the present study, the NRI 

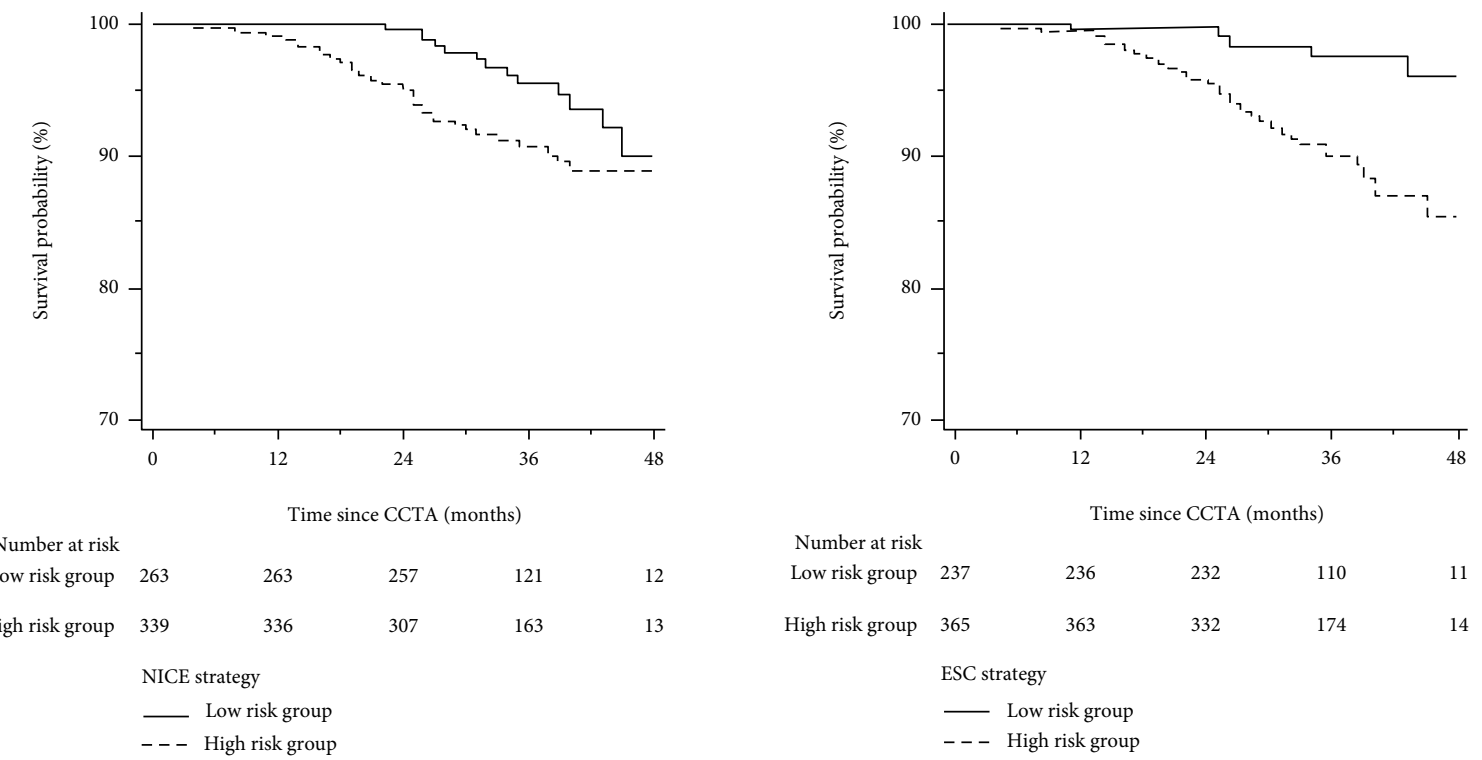

FIgure 1: Cumulative survival probability from MACE in low- and high-risk groups determined by the NICE and ESC strategy. Abbreviations as in Table 3.

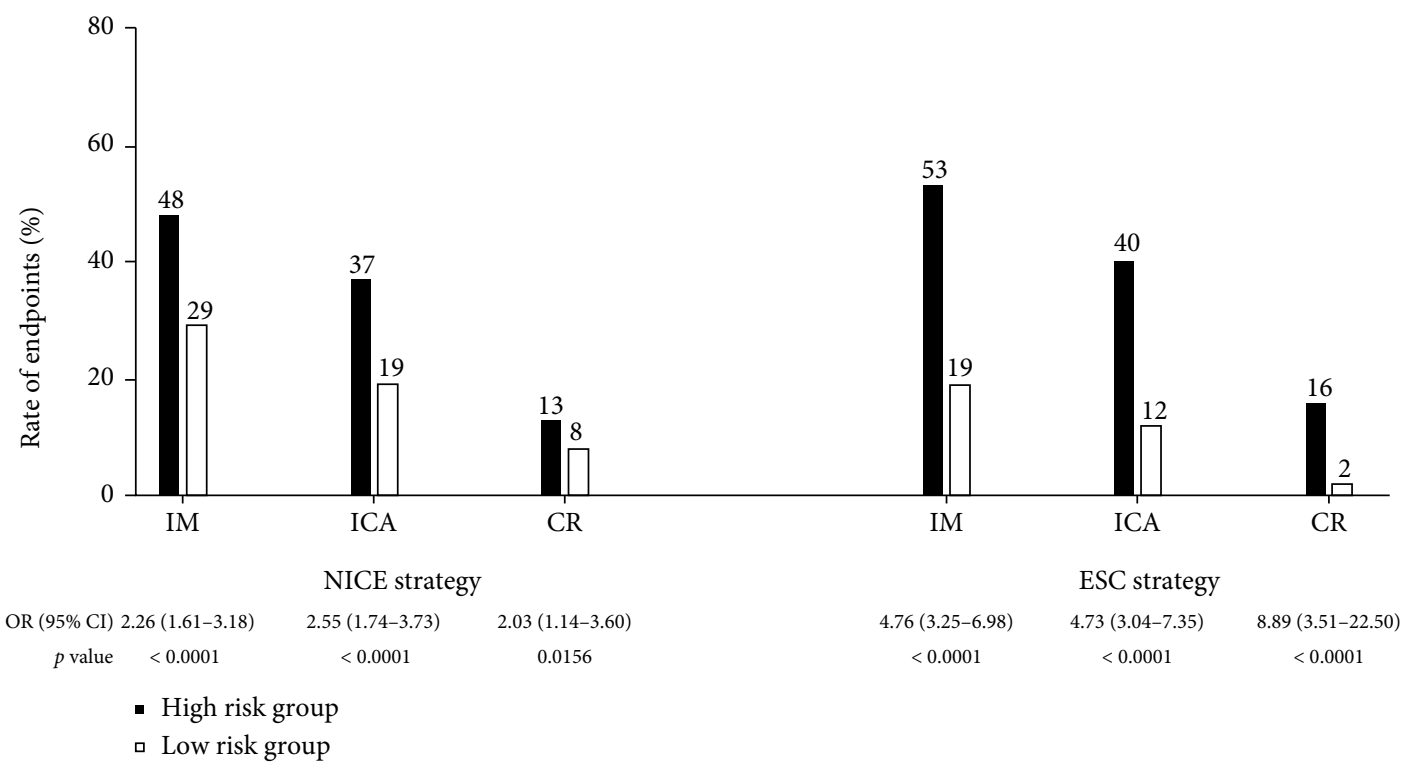

FIGURE 2: Rates for secondary endpoints in low- and high-risk groups determined by the NICE and ESC strategy. ICA: invasive coronary angiography; IM: increase of medication; CR: coronary revascularization; other abbreviations as in Table 3.

was negative and the association between study endpoints and risk groups was attenuated.

Both the ESC-PTP estimator and RF-CL model were developed in the most contemporary SCP cohorts and indicative of the best performance to predict obstructive CAD and MACE in general SCP patients [14-16, 24], which were compliant with the modest AUC for the ESC-PTP estimator and positive NRI comparing the RF-CL model to the NICE strategy in the present study. In addition, the RF-CL model has also taken the interaction effect between symptom and DM into account [24]. As a result, the ESC strategy based on the sequential amalgamation of the ESC-PTP estimator and RF-CL model demonstrated superiority in terms of the diagnosis for obstructive CAD, prediction of MACE, and use of downstream diagnostic and therapeutic interventions in patients with DM and SCP.

As illustrated in Figure 3, the ESC-PTP estimator overestimated the probability of obstructive CAD in patients with ESC-PTP $>15 \%$. The overestimation may not change the further clinical management, because all patients with ESC -PTP $>15 \%$ should be referred to CIT according to the ESC strategy [11]. On the contrary, the underestimation for probability of obstructive CAD in patients with ESCPTP between $5 \%$ and $15 \%$ may result in a significant number of missing referrals for CIT. To provide a more indepth and comprehensive insight into the risk assessment 


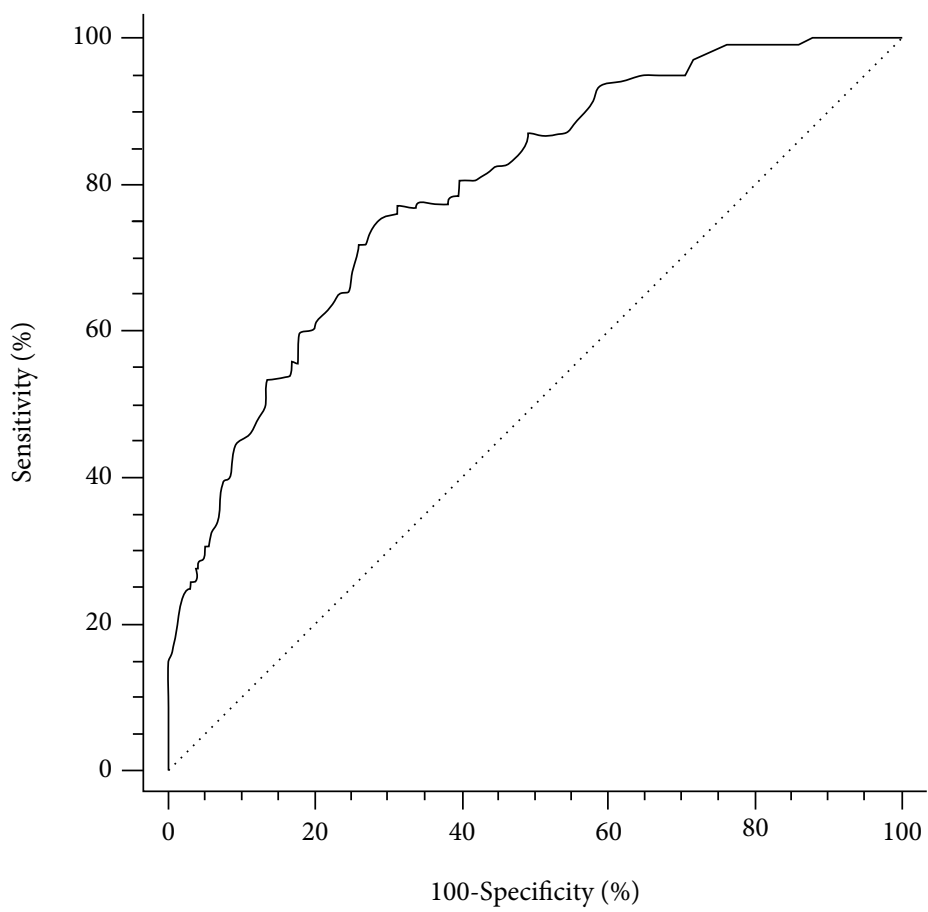

FIGURE 3: ROC curves for the ESC-PTP estimator to predict obstructive CAD. ROC: receiver operating characteristic; other abbreviations as in Table 4.

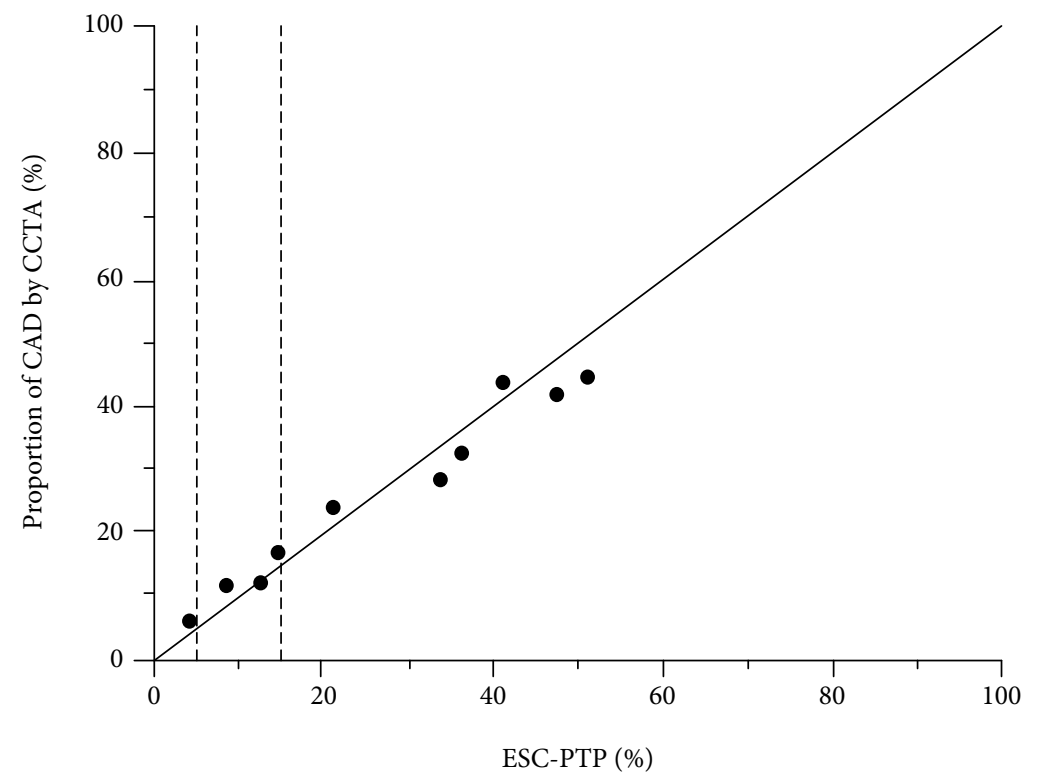

FIGURE 4: Comparison of ESC-PTP and proportion of obstructive CAD on CCTA by deciles of ESC-PTP. The area between two dotted lines represents ESC-PTP between 5\% and 15\%. CCTA: coronary computed tomographic angiography. Other abbreviations as in Figure 3.

for patients with borderline ESC-PTP, we also conducted the comparison between the RF-CL model and NICE strategy in patients with ESC-PTP between 5\% and 15\% (Table 4) and among the RF-CL model, ESC strategy, and NICE strategy in ESC-PTP below 5\% and above 15\% (Table 5), respectively. Taking all these into consideration, when comparing the ESC strategy to the NICE strategy, the application of the RF-CL model obviously ameliorated the underestima- tion of risk in 208 patients with borderline ESC-PTP and the total reclassification of 59 positive patients should be principally $(61.02 \%, 36 / 59)$ attributed to this application.

More importantly, the RF-CL model incorporated symptom assessment plus risk factors which were easily accessible in daily clinical practice. Thus, one additional collection of information for risk factors in every $602 / 208 \approx 3 \mathrm{DM}$ patients and one avoidance of missing referrals for CIT 
TABLE 3: Reclassification table comparing the ESC strategy to the NICE strategy.

\begin{tabular}{|c|c|c|c|c|c|c|c|}
\hline & \multicolumn{2}{|c|}{$\begin{array}{l}\text { Risk groups by } \\
\text { ESC strategy }\end{array}$} & \multirow{2}{*}{ Total } & \multicolumn{2}{|c|}{ Reclassification } & \multirow{2}{*}{ NRI } & \multirow{2}{*}{$p$} \\
\hline & Low & High & & Up & Down & & \\
\hline \multicolumn{8}{|c|}{ Risk groups by NICE strategy } \\
\hline Negative patients & & & & $2.42 \%$ & $10.57 \%$ & $27.71 \%$ & $<0.0001$ \\
\hline Low & 190 & 8 & 198 & & & & \\
\hline High & 35 & 98 & 133 & & & & \\
\hline Total & 225 & 106 & 331 & & & & \\
\hline Positive patients & & & & $21.77 \%$ & $2.21 \%$ & & \\
\hline Low & 6 & 59 & 65 & & & & \\
\hline High & 6 & 200 & 206 & & & & \\
\hline Total & 12 & 259 & 271 & & & & \\
\hline
\end{tabular}

CAD: coronary artery disease; NICE strategy: 2016 National Institute of Health and Care Excellence guideline-determined risk assessment strategy; ESC strategy: 2019 European Society of Cardiology guideline-determined risk assessment strategy.

TABLE 4: Reclassification table comparing the RF-CL model to the NICE strategy in patients with borderline ESC-PTP.

\begin{tabular}{|c|c|c|c|c|c|c|c|}
\hline & \multicolumn{2}{|c|}{$\begin{array}{l}\text { Risk groups by } \\
\text { RF-CL model }\end{array}$} & \multirow{2}{*}{ Total } & \multicolumn{2}{|c|}{ Reclassification } & \multirow[t]{2}{*}{ NRI } & \multirow[t]{2}{*}{$p$} \\
\hline & Low & High & & Up & Down & & \\
\hline \multicolumn{8}{|c|}{ Risk groups by NICE strategy } \\
\hline Negative patients & & & & $2.68 \%$ & $12.50 \%$ & $42.11 \%$ & $<0.0001$ \\
\hline Low & 65 & 3 & 68 & & & & \\
\hline High & 14 & 30 & 44 & & & & \\
\hline Total & 79 & 33 & 112 & & & & \\
\hline Positive patients & & & & $37.50 \%$ & $5.21 \%$ & & \\
\hline Low & 3 & 36 & 39 & & & & \\
\hline High & 5 & 52 & 57 & & & & \\
\hline Total & 8 & 88 & 96 & & & & \\
\hline
\end{tabular}

RF-CL: risk factor-weighted clinical likelihood; ESC-PTP: 2019 European Society of Cardiology guideline-determined pretest probability; other abbreviations as in Table 3.

corresponding to $208 /(36-5) \approx 7$ additional collection of information for risk factors made the ESC strategy more efficient. As mentioned above, the optimal risk assessment strategy to guide the screening of CAD in patients with DM and SCP has great clinical importance. In this context, instead of the NICE strategy which mainly focused on symptom evaluation, the ESC strategy which sequentially incorporated different PTP models might provide more feasible identification of DM patients who may derive maximal benefit from further CIT.

4.1. Limitations. Although this is the first study to evaluate proposed risk assessment strategies for patients with DM and SCP, several issues merit consideration. First, this study was an observational cohort. Clinical management of patients with DM and SCP before and after CCTA relied on a local physician. More details about medical therapy and CR during follow-up were not available. Thus, whether the ESC strategy will lead to more appropriate decisionmaking of downstream referral and better clinical outcomes for patients with DM and SCP needs to be addressed in further studies, such as randomized controlled trials. Second, using data from the PROMISE cohort, Fordyce et al. developed a new tool to identify patients deriving minimal value from CIT [33]. Although the PROMISE minimal risk tool [34] has been externally validated, no recent clinical guideline recommends it as the risk assessment tool for patients with SCP. Third, this analysis focused on the presence of obstructive CAD documented by CCTA. Previous studies have demonstrated that CCTA had a high negative predictive value compared with ICA $[35,36]$. So CCTA could offer robust reassurance for both strategies to exclude obstructive CAD. Moreover, we defined unassessable segments as positive ones based on current guideline recommendations in which further testing should be referred for nonconclusive CCTA. Fourth, a coronary artery calcium score $[20,37]$ and high-sensitivity cardiac troponin $[38,39]$ have shown the potential to improve risk assessment for patients with DM and SCP. However, additional imaging or blood testing is needed for the two attractive biomarkers, and their costeffectiveness warrants further evaluation. Fifth, as the majority of patients had missing data about other ECG changes such as $Q$ wave, we only analyzed ST-T changes. This could reduce the size of the high-risk group in the NICE strategy, 
TABLE 5: Reclassification table comparing the RF-CL model, NICE strategy, and ESC strategy in patients with ESC-PTP below 5\% and above $15 \%$.

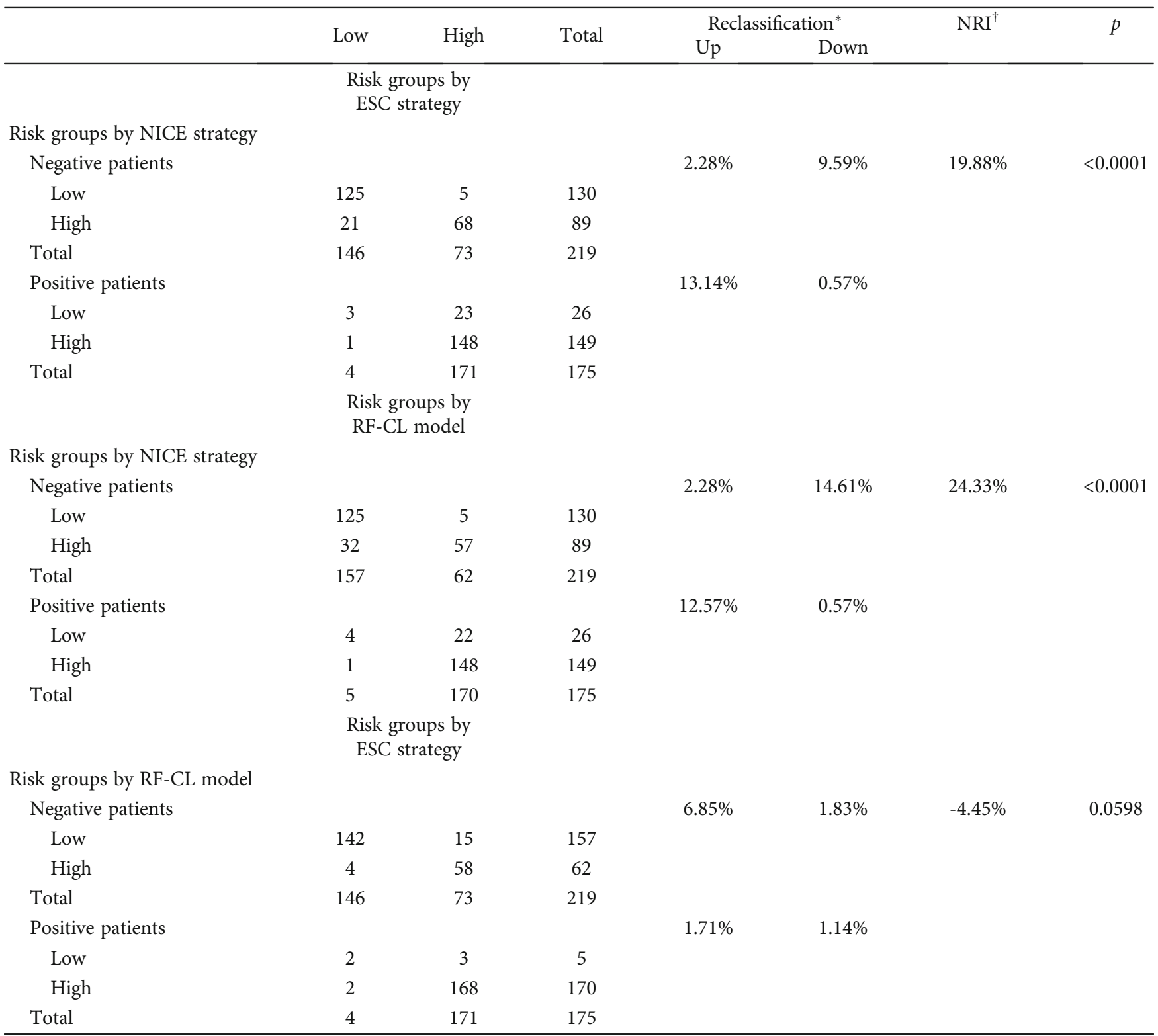

Abbreviations as in Table 3.

especially in DM patients for whom the silent ischemia is common [9, 31, 32]. Finally, more researches about the performance of different strategies in different subgroups of age and sex were needed in the future.

\section{Conclusions}

Compared to the symptom-focused strategy, the ESC strategy based on PTP estimation seemed to be associated with greater efficiency in identifying high-risk individuals who may derive maximum benefit from further CIT in patients with DM and SCP. This superiority should be dominantly ascribed to the application of the RF-CL model in borderline patients. For more accurate and convenient risk assessment in patients with DM and SCP suggestive of obstructive $\mathrm{CAD}$, further investigations with comprehensive and rigorous design are needed.

\section{Data Availability}

The data used to support the findings of this study are available from the corresponding author upon request.

\section{Conflicts of Interest}

No conflicting relationship exists for any author. 


\section{Authors' Contributions}

Jia Zhao, Shuo Wang, and Pengyu Zhao contributed equally to this work.

\section{Acknowledgments}

This study was supported by the Tianjin Key Research Program of Traditional Chinese Medicine (2022001), National Key Research and Development Program of China (2016YFC1301100 and 2016YFC1300300), and Research Program of Tianjin Chest Hospital (2018XKC10).

\section{References}

[1] W. Ling, Y. Huang, Y. M. Huang, R. R. Fan, Y. Sui, and H. L. Zhao, "Global trend of diabetes mortality attributed to vascular complications, 2000-2016," Cardiovascular Diabetology, vol. 19, no. 1, p. 182, 2020.

[2] A. Sharma, N. K. Sekaran, A. Coles et al., "Impact of diabetes mellitus on the evaluation of stable chest pain patients: insights from the PROMISE (prospective multicenter imaging study for evaluation of chest pain) trial," Journal of the American Heart Association, vol. 6, no. 11, 2017.

[3] C. Junghans, N. Sekhri, M. J. Zaman, H. Hemingway, G. S. Feder, and A. Timmis, "Atypical chest pain in diabetic patients with suspected stable angina: impact on diagnosis and coronary outcomes," European Heart Journal-Quality of Care and Clinical Outcomes, vol. 1, no. 1, pp. 37-43, 2015.

[4] J. Narula, Y. Chandrashekhar, A. Ahmadi et al., "SCCT 2021 expert consensus document on coronary computed tomographic angiography: a report of the Society of Cardiovascular Computed Tomography," Journal of Cardiovascular Computed Tomography, vol. 15, no. 3, pp. 192-217, 2021.

[5] A. Timmis and C. A. Roobottom, "National Institute for Health and Care Excellence updates the stable chest pain guideline with radical changes to the diagnostic paradigm," Heart, vol. 103, no. 13, pp. 982-986, 2017.

[6] P. C. Cremer and S. E. Nissen, "The National Institute for Health and Care Excellence update for stable chest pain: poorly reasoned and risky for patients," Heart, vol. 103, no. 13, pp. 972-974, 2017.

[7] J. E. Udelson and U. Hoffmann, "The United Kingdom's National Institute for Health and Care Excellence guideline on chest pain of recent onset: a United States perspective," Journal of Nuclear Cardiology, vol. 24, no. 5, pp. 1535-1539, 2017.

[8] H. S. Hecht, L. Shaw, Y. S. Chandrashekhar, J. J. Bax, and J. Narula, "Should NICE guidelines be universally accepted for the evaluation of stable coronary disease? A debate," European heart journal, vol. 40, no. 18, pp. 1440-1453, 2019.

[9] P. G. Jørgensen, M. T. Jensen, R. Mogelvang et al., “Abnormal echocardiography in patients with type 2 diabetes and relation to symptoms and clinical characteristics," Diabetes \& Vascular Disease Research, vol. 13, no. 5, pp. 321-330, 2016.

[10] J. Knuuti, W. Wijns, A. Saraste et al., "2019 ESC guidelines for the diagnosis and management of chronic coronary syndromes," European Heart Journal, vol. 41, no. 3, pp. 407477, 2020.

[11] A. Saraste, E. Barbato, D. Capodanno et al., "Imaging in ESC clinical guidelines: chronic coronary syndromes," European
Heart Journal Cardiovascular Imaging, vol. 20, no. 11, pp. 1187-1197, 2019.

[12] P. D. Adamson, D. E. Newby, C. L. Hill, A. Coles, P. S. Douglas, and C. B. Fordyce, "Comparison of international guidelines for assessment of suspected stable angina: insights from the PROMISE and SCOT-HEART," JACC. Cardiovascular Imaging, vol. 11, no. 9, pp. 1301-1310, 2018.

[13] P. D. Adamson, A. Hunter, M. C. Williams et al., "Diagnostic and prognostic benefits of computed tomography coronary angiography using the 2016 National Institute for Health and Care Excellence guidance within a randomised trial," Heart, vol. 104, no. 3, pp. 207-214, 2018.

[14] R. Bing, T. Singh, M. R. Dweck et al., "Validation of European Society of Cardiology pre-test probabilities for obstructive coronary artery disease in suspected stable angina," European Heart Journal-Quality of Care and Clinical Outcomes, vol. 6, no. 4, pp. 293-300, 2020.

[15] L. D. Rasmussen, L. Nissen, J. Westra et al., "Validation and update of the minimal risk tool in patients suspected of chronic coronary syndrome," The International Journal of Cardiovascular Imaging, vol. 37, no. 2, pp. 699-706, 2021.

[16] S. Winther, S. E. Schmidt, L. D. Rasmussen et al., "Validation of the European Society of Cardiology pre-test probability model for obstructive coronary artery disease," European Heart Journal, vol. 42, no. 14, pp. 1401-1411, 2021.

[17] J. B. Muhlestein, D. L. Lappé, J. A. Lima et al., "Effect of screening for coronary artery disease using CT angiography on mortality and cardiac events in high-risk patients with diabetes: the FACTOR-64 randomized clinical trial," Journal of the American Medical Association, vol. 312, no. 21, pp. 2234-2243, 2014.

[18] Y. Zhang, Y. Liu, H. Zhang, and J. Zhou, "Impact of sexspecific differences in calculating the pretest probability of obstructive coronary artery disease in symptomatic patients: a coronary computed tomographic angiography study," Coronary Artery Disease, vol. 30, no. 2, pp. 124-130, 2019.

[19] J. Zhou, Y. Chen, Y. Zhang et al., "Epicardial fat volume improves the prediction of obstructive coronary artery disease above traditional risk factors and coronary calcium score," Circulation. Cardiovascular Imaging, vol. 12, no. 1, p. e008002, 2019.

[20] J. Zhou, Y. Liu, L. Huang et al., "Validation and comparison of four models to calculate pretest probability of obstructive coronary artery disease in a Chinese population: a coronary computed tomographic angiography study," Journal of Cardiovascular Computed Tomography, vol. 11, no. 4, pp. 317-323, 2017.

[21] J. Zhou, J. Zhao, Z. Li et al., "Coronary calcification improves the estimation for clinical likelihood of obstructive coronary artery disease and avoids unnecessary testing in patients with borderline pretest probability," European Journal of Preventive Cardiology, 2021.

[22] G. A. Diamond and J. S. Forrester, "Analysis of probability as an aid in the clinical diagnosis of coronary-artery disease," New England Journal of Medicine, vol. 300, no. 24, pp. 13501358, 1979.

[23] A. S. Levey, L. A. Stevens, C. H. Schmid et al., "A new equation to estimate glomerular filtration rate," Annals of Internal Medicine, vol. 150, no. 9, pp. 604-612, 2009.

[24] S. Winther, S. E. Schmidt, T. Mayrhofer et al., "Incorporating coronary calcification into pre-test assessment of the likelihood of coronary artery disease," Journal of the American College of Cardiology, vol. 76, no. 21, pp. 2421-2432, 2020. 
[25] J. Leipsic, S. Abbara, S. Achenbach et al., "SCCT guidelines for the interpretation and reporting of coronary CT angiography: a report of the Society of Cardiovascular Computed Tomography Guidelines Committee," Journal of Cardiovascular Computed Tomography, vol. 8, no. 5, pp. 342-358, 2014.

[26] R. C. Cury, S. Abbara, S. Achenbach et al., "CAD-RADS ${ }^{\mathrm{TM}}$ Coronary Artery Disease - Reporting and Data System. An expert consensus document of the Society of Cardiovascular Computed Tomography (SCCT), the American College of Radiology (ACR) and the North American Society for Cardiovascular Imaging (NASCI). Endorsed by the American College of Cardiology," Journal of Cardiovascular Computed Tomography, vol. 10, no. 4, pp. 269-281, 2016.

[27] K. Thygesen, J. S. Alpert, A. S. Jaffe et al., "Fourth universal definition of myocardial infarction (2018)," Journal of the American College of Cardiology, vol. 72, no. 18, pp. 22312264, 2018.

[28] G. S. Collins, J. B. Reitsma, D. G. Altman, K. G. Moons, and Group T, "Transparent reporting of a multivariable prediction model for individual prognosis or diagnosis (TRIPOD)," The TRIPOD Group. Circulation, vol. 131, no. 2, pp. 211-219, 2015.

[29] A. C. Alba, T. Agoritsas, M. Walsh et al., "Discrimination and calibration of clinical prediction models," Journal of the American Medical Association, vol. 318, no. 14, pp. 1377-1384, 2017.

[30] F. Turrini, S. Scarlini, C. Mannucci et al., "Does coronary atherosclerosis deserve to be diagnosed early in diabetic patients? The DADDY-D trial. Screening diabetic patients for unknown coronary disease," European Journal of Internal Medicine, vol. 26, no. 6, pp. 407-413, 2015.

[31] J. S. Yun, Y. M. Park, S. A. Cha, Y. B. Ahn, and S. H. Ko, "Progression of cardiovascular autonomic neuropathy and cardiovascular disease in type 2 diabetes," Cardiovascular diabetology, vol. 17, no. 1, p. 109, 2018.

[32] V. Spallone, "Update on the impact, diagnosis and management of cardiovascular autonomic neuropathy in diabetes: what is defined, what is new, and what is unmet," Diabetes and Metabolism Journal, vol. 43, no. 1, pp. 3-30, 2019.

[33] C. B. Fordyce, P. S. Douglas, R. S. Roberts et al., "Identification of patients with stable chest pain deriving minimal value from noninvasive testing: the PROMISE minimal-risk tool, a secondary analysis of a randomized clinical trial," JAMA Cardiology, vol. 2, no. 4, pp. 400-408, 2017.

[34] P. D. Adamson, C. B. Fordyce, D. A. McAllister, J. E. Udelson, P. S. Douglas, and D. E. Newby, "Identification of patients with stable chest pain deriving minimal value from coronary computed tomography angiography: an external validation of the PROMISE minimal-risk tool," International Journal of Cardiology, vol. 252, pp. 31-34, 2018.

[35] R. Haase, P. Schlattmann, P. Gueret et al., "Diagnosis of obstructive coronary artery disease using computed tomography angiography in patients with stable chest pain depending on clinical probability and in clinically important subgroups: meta-analysis of individual patient data," BMJ, vol. 365, p. 11945, 2019.

[36] J. Knuuti, H. Ballo, L. E. Juarez-Orozco et al., "The performance of non-invasive tests to rule-in and rule-out significant coronary artery stenosis in patients with stable angina: a metaanalysis focused on post-test disease probability," European Heart Journal, vol. 39, no. 35, pp. 3322-3330, 2018.
[37] T. S. Genders, E. W. Steyerberg, M. G. Hunink et al., "Prediction model to estimate presence of coronary artery disease: retrospective pooled analysis of existing cohorts," BMJ, vol. 344, p. e3485, 2012.

[38] J. L. Januzzi Jr., S. Suchindran, U. Hoffmann et al., "Singlemolecule hsTnI and short-term risk in stable patients with chest pain," Journal of the American College of Cardiology, vol. 73, no. 3, pp. 251-260, 2019.

[39] B. M. Everett, M. M. Brooks, H. E. Vlachos, B. R. Chaitman, R. L. Frye, and D. L. Bhatt, "Troponin and cardiac events in stable ischemic heart disease and diabetes," The New England Journal of Medicine, vol. 373, no. 7, pp. 610-620, 2015. 